\title{
Originales
}

\section{Deterioro agudo de la función renal en una Unidad Coronaria en España}

\author{
D. MARTía , C. ESCOBAR ${ }^{a}$, N. DEDIEU ${ }^{b}$, F. LIAÑO ${ }^{c}$, M. JIMÉNEZ MENA ${ }^{a}$ Y E. ASÍN ${ }^{a}$ \\ aServicio de Cardiología. ' Servicio de Cardiología Pediátrica. `Servicio de Nefrología. Hospital Ramón y Cajal. \\ Madrid. España.
}

Objetivo. Valorar la incidencia y el valor pronóstico del deterioro agudo de la función renal (DAFR) en pacientes cardiológicos agudos.

Ámbito. Unidad Coronaria de un hospital universitario terciario.

Diseño. Estudio retrospectivo.

Pacientes. Cien pacientes consecutivos ingresados durante 2004.

Intervenciones. No hubo intervenciones aleatorizadas. Los procedimientos diagnósticos y terapéuticos se realizaron de acuerdo con protocolos locales y las Guías de Práctica Clínica en uso.

Variables principales. El objetivo primario del estudio fue analizar la incidencia de DAFR y su relación con la mortalidad por cualquier causa durante el ingreso. Se definió DAFR como el aumento de la creatinina sérica respecto a la basal mayor de $0,5 \mathrm{mg} / \mathrm{dl}$ y/o mayor del $50 \%$.

Resultados. La incidencia de DAFR fue del $26 \%$, con un incremento medio de la creatinina sérica de 1,5 $\pm 0,9 \mathrm{mg} / \mathrm{dl}$. El DAFR se asoció de forma significativa con la edad y los antecedentes de hipertensión e insuficiencia renal crónica. Los pacientes con DAFR tuvieron un curso más complicado, mayores estancias hospitalarias y recibieron menos cateterismos. El empeoramiento agudo de la función renal se asoció con mayor mortalidad hospitalaria $(33 \%$ frente a $6 \%, p=0,002)$.

Conclusiones. EI DAFR es frecuente en el paciente cardiópata agudo y su presencia se asocia con alta mortalidad.

Correspondencia: Dr. D. Martí Sánchez.

Servicio de Cardiología. Hospital Ramón y Cajal.

Ctra. Colmenar Viejo, km 9,100.

28034 Madrid. España.

Correo electrónico: docalcala@hotmail.com

Manuscrito aceptado el 1-X-2007.
PALABRAS CLAVE: insuficiencia renal, Unidad Coronaria, muerte.

\section{ACUTE RENAL FUNCTION DETERIORATION IN A CORONARY UNIT IN SPAIN}

Objective. To assess the incidence and prognostic value of acute renal function deterioration (ARFD) in patients with acute heart disease.

Setting. Coronary Care Unit in a tertiary university hospital.

Design. Retrospective study.

Participants. One hundred consecutive patients admitted during 2004.

Interventions. No randomized interventions were done. Diagnostic and therapeutic procedures were performed according to local protocols and current Clinical Practice Guidelines.

Primary variables. The primary aim of the study was to analyze the incidence of acute renal function deterioration and its effect in mortality during hospitalization. ARFD was defined as the increase of serum creatinine by $0.5 \mathrm{mg} / \mathrm{dl}$ and/or by $50 \%$ over baseline.

Results. Incidence of ARFD was $26 \%$, with a mean increase of serum creatinine of $1.5 \pm 0.9$ $\mathrm{mg} / \mathrm{dl}$. ARFD was significantly associated with age, background of hypertension and chronic kidney disease. Patients with ARFD had a more complicated course, longer hospitalizations, and received fewer catheterisms. Acute renal function deterioration was associated with higher mortality during hospitalization $(33 \%$ versus $6 \%$,

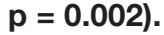

Conclusions. Acute renal function deterioration is frequent in patients with acute heart disease and its presence is linked with higher mortality.

KEY WORDS: kidney failure, Coronary Unit, death. 


\section{INTRODUCCIÓN}

Las enfermedades cardiovasculares constituyen la primera causa de muerte en los países industrializados, y son responsables de más de la tercera parte de las defunciones en nuestro país ${ }^{1}$. La patología cardiaca es más frecuente y de peor pronóstico en los pacientes con nefropatía ${ }^{2-4}$. Es responsable del $50 \%$ de las muertes de los pacientes con insuficiencia renal crónica y motiva un tercio de los ingresos hospitalarios de los pacientes en diálisis. Más de la mitad de los pacientes con insuficiencia renal terminal que sufren un evento coronario agudo fallece durante el siguiente año $0^{5}$ Los pacientes nefrópatas que se someten a procedimientos de revascularización percutánea tienen mayor riesgo de reestenosis ${ }^{6}$ y de trombosis del stent ${ }^{7}$; además, la revascularización quirúrgica conlleva una mayor morbimortalidad que en la población general ${ }^{8}$. La nefropatía también es particularmente frecuente en los pacientes cardiológicos. Aproximadamente el 10\% de los pacientes con enfermedad coronaria tienen insuficiencia renal crónica, y la insuficiencia cardiaca es una de las principales causas de fracaso renal agudo 9 . Diversos fármacos y procedimientos de uso habitual en cardiología pueden deteriorar la función renal ${ }^{10}$. La insuficiencia renal constituye un factor de riesgo independiente de mortalidad en pacientes con insuficiencia cardiaca aguda o crónica, en pacientes con síndrome coronario agudo (SCA) y en los sometidos a técnicas de revascularización percutánea o quirúrgica ${ }^{10-15}$.

Aunque varios estudios extranjeros abordan el deterioro de la función renal en los pacientes cardiológicos agudos, existe escasa información sobre la incidencia, el manejo y el pronóstico de este tipo de pacientes en nuestro medio.

\section{PACIENTES Y MÉTODOS}

Para la realización del estudio se incluyeron los 100 primeros pacientes consecutivos ingresados en la Unidad Coronaria del Hospital Universitario Ramón y Cajal durante el año 2004. Se analizaron las características basales de los pacientes, factores de riesgo cardiovascular y condiciones clínicas asociadas. Los procedimientos diagnósticos y terapéuticos se realizaron siguiendo los protocolos locales y las Guías de Práctica Clínica de la Sociedad Española de Cardiología y la Sociedad Europea de Cardiología.

El parámetro principal de valoración fue la mortalidad por cualquier causa durante el ingreso. Se analizaron también las estancias totales y en la Unidad de Cuidados Intensivos, y la necesidad de técnicas terapéuticas agresivas.

Se definió deterioro agudo de la función renal (DAFR) como el aumento de la creatinina sérica respecto a la basal mayor de $0,5 \mathrm{mg} / \mathrm{dl}$ y/o mayor del $50 \%$. Se determinaron los productos nitrogenados diariamente durante el ingreso en la Unidad de Cuidados Agudos, cada 48 horas durante el ingreso en la Unidad de Cuidados Intermedios y a criterio del médico responsable durante el ingreso en planta de
TABLA 1. Características basales de los pacientes $(n=91)$

\begin{tabular}{|lc|}
\hline Edad & $66 \pm 13$ años \\
Sexo & $63 \%$ varones \\
& $37 \%$ mujeres \\
Hipertensión arterial & $58 \%$ \\
Diabetes mellitus & $34 \%$ \\
Dislipemia & $27 \%$ \\
Antecedentes de tabaquismo & $42 \%$ \\
$\geq 2$ factores de riesgo cardiovascular & $53 \%$ \\
Enfermedad arterial periférica & $19 \%$ \\
Cardiopatía previa & \\
Ninguna conocida & $62 \%$ \\
Isquémica & $28 \%$ \\
Valvular & $3 \%$ \\
Miocardiopatía dilatada & $3 \%$ \\
Otra & $3 \%$ \\
Insuficiencia renal crónica conocida & $18 \%$ \\
\hline
\end{tabular}

hospitalización. Se tomaron analíticas adicionales siempre que se consideró oportuno por la situación clínica del paciente. El diseño del estudio fue aprobado por el comité de Ética local.

Las pruebas estadísticas se realizaron dependiendo de la naturaleza de las variables. El estudio de la relación de variables categóricas se realizó mediante la prueba de Chi cuadrado (en el caso de que más del $20 \%$ de las celdas tuviera una frecuencia esperada menor a 5 se utilizó la prueba exacta de Fisher). La comparación de variables continuas entre grupos de pacientes se realizó mediante la prueba de la «t» de Student. Todos los cálculos estadísticos fueron realizados con el paquete de software SPSS versión 12.0 (Chicago, IL).

\section{RESULTADOS}

Se incluyeron un total de 100 pacientes, 9 de los cuales fueron excluidos para el análisis final: 4 de ellos por continuar su seguimiento en otro centro, lo que impidió obtener los datos de forma completa; dos pacientes por haber sido ingresados de forma programada para la realización de una cardioversión eléctrica por fibrilación auricular; y tres por ser ingresos por causa no cardiológica.

La población final del estudio constó de 91 pacientes, cuyas características basales se muestran en la tabla 1. La edad media de los pacientes ingresados en la Unidad Coronaria fue de $66 \pm 13$ años, con un $63 \%$ de varones. El factor de riesgo cardiovascular más prevalente fue la hipertensión arterial, seguido de la historia de tabaquismo. El 53\% de los pacientes presentaba al menos dos factores de riesgo. El ingreso en la Unidad fue el comienzo cardiológico en el $62 \%$ de los enfermos.

El motivo de ingreso (fig. 1) más frecuente fue el síndrome coronario agudo sin elevación del segmento ST (SCASEST) (44\%), seguido del síndrome coronario agudo con elevación del segmento ST (SCACEST) $(26,4 \%)$, insuficiencia cardiaca $(16,5 \%)$ y otros motivos $(13,2 \%)$, como miocarditis o arritmias graves. 


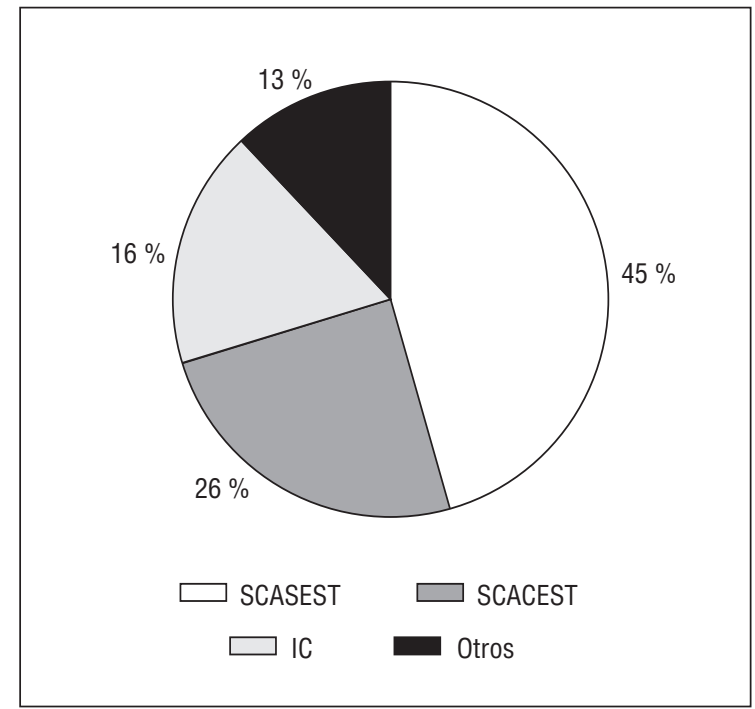

Figura 1. Diagnóstico de ingreso en la Unidad Coronaria. SCACEST: síndrome coronario agudo con elevación del segmento ST; SCASEST: síndrome coronario agudo sin elevación del segmento ST; IC: insuficiencia cardiaca.

La estancia media en la Unidad Coronaria fue de $5,6 \pm$ 4,6 días, y la hospitalaria total de 15,2 $\pm 9,3$ días. Se practicó coronariografía diagnóstica en el $82 \%$ de los SCA, revascularización percutánea en el $47 \%$ y revascularización quirúrgica en el $6 \%$. En el $66 \%$ de los pacientes con SCACEST se realizó angioplastia primaria, y el $24 \%$ se presentó en el hospital con más de 12 horas de evolución y sin dolor, por lo que no se indicó reperfusión urgente. En ningún caso se hizo fibrinólisis. Se obtuvieron datos de función sistólica por ventriculografía o ecocardiografía en el $90 \%$ de los pacientes. De ellos, el $72 \%$ presentó una fracción de eyección del ventrículo izquierdo mayor al $50 \%$, el $10 \%$ entre 40 y 50 , el $11 \%$ entre 30 y 40 y el $7 \%$ menor del $30 \%$. El $3 \%$ de los pacientes precisó balón de contrapulsación intraaórtico (BIAC), el $8 \%$ intubación orotraqueal (IOT) y ventilación mecánica y el $2 \%$ terapia renal sustitutiva.

El $13 \%$ de los pacientes fallecieron durante el ingreso. La insuficiencia cardiaca fue la primera causa de muerte, ya que fue responsable del $66 \%$ de ellas.

La incidencia global de DAFR fue del $26 \%$, con un aumento medio de la creatinina sérica respecto a la basal de $1,5 \pm 0,9 \mathrm{mg} / \mathrm{dl}$.

Los pacientes con DAFR (tabla 2) fueron significativamente mayores, más hipertensos y con más nefropatía previa. Además, tendieron a presentar con más frecuencia múltiples factores de riesgo, diabetes, arteriopatía periférica y antecedentes de enfermedad cardiaca. Como se puede observar en la tabla 3, mostraron tendencia a ingresar con más frecuencia por SCASEST o insuficiencia cardiaca. La presencia de DAFR se asoció con estancias hospitalarias totales significativamente más largas (18,7 frente a 13,9 días, $\mathrm{p}=0,03)$, pero no con estancias mayores en la Unidad Coronaria (7,0 frente a 5,1 días, $\mathrm{p}=0,08)$. Estos pacientes presentaron peor evolución, con mayor necesidad de BIAC, IOT y técnicas sustitutivas renales analizados conjuntamente $(p=0,046)$. Los pacientes que ingresaron por SCA y presentaron DAFR recibieron significativamente menos cateterismos $(\mathrm{p}=0,038)$ y técnicas intervencionistas percutáneas $(\mathrm{p}=0,038)$.

Los pacientes con DAFR recibieron al alta menos inhibidores del sistema renina angiotensina $(p=0,01)$ y más diuréticos $(\mathrm{p}=0,007)$. No hubo diferencias en la prescripción de espironolactona.

El DAFR se asoció a una mortalidad por cualquier causa significativamente mayor durante el ingreso (riesgo relativo 5,5, intervalo de confianza al 95\%, 1,8-16,9, $\mathrm{p}=0,002$ ) (fig. 2).

\section{DISCUSIÓN}

En nuestro estudio se incluyeron pacientes ingresados en la Unidad Coronaria durante el año 2004, por lo que se empleó una definición de DAFR previa

TABLA 2. Características de los pacientes en función de la ausencia o presencia de deterioro agudo de la función renal

\begin{tabular}{|c|c|c|c|}
\hline & No DAFR $(\mathrm{n}=67)$ & Sí DAFR ( $\mathrm{n}=24)$ & $\mathrm{p}$ \\
\hline Edad & $63 \pm 14$ años & $73 \pm 6$ años & 0,001 \\
\hline \multirow{2}{*}{ Sexo } & $64 \%$ varones & $58 \%$ varones & 0,61 \\
\hline & $36 \%$ mujeres & $41 \%$ mujeres & \\
\hline Hipertensión arterial & $50 \%$ & $79 \%$ & 0,015 \\
\hline Diabetes & $32 \%$ & $37 \%$ & 0,67 \\
\hline Dislipemia & $27 \%$ & $29 \%$ & 0,82 \\
\hline Historia de tabaquismo & $52 \%$ & $16 \%$ & 0,003 \\
\hline$\geq 2 \mathrm{FRCV}$ & $55 \%$ & $50 \%$ & 0,66 \\
\hline Arteriopatía periférica & $16 \%$ & $29 \%$ & 0,26 \\
\hline Cardiopatía previa & & & 0,13 \\
\hline Ninguna & $67 \%$ & $50 \%$ & \\
\hline Isquémica & $26 \%$ & $33 \%$ & \\
\hline Valvular & $1,50 \%$ & $8 \%$ & \\
\hline Miocardiopatía dilatada & $1,50 \%$ & $4 \%$ & \\
\hline Otra & $3 \%$ & $4 \%$ & \\
\hline Insuficiencia renal crónica & $9 \%$ & $45 \%$ & $<0,001$ \\
\hline
\end{tabular}

DAFR: deterioro agudo de la función renal; FRCV: factores de riesgo cardiovascular. 
TABLA 3. Curso durante el ingreso en función del deterioro agudo de la función renal

\begin{tabular}{|c|c|c|c|}
\hline & No DAFR $(\mathrm{n}=67)$ & Sí DAFR $(n=24)$ & $\mathrm{p}$ \\
\hline Diagnóstico principal & & & 0,058 \\
\hline SCASEST & $41 \%$ & $50 \%$ & \\
\hline SCACEST & $32 \%$ & $8 \%$ & \\
\hline IC & $12 \%$ & $29 \%$ & \\
\hline \multicolumn{4}{|l|}{ Estancia media } \\
\hline UC & $5,1 \pm 3,6$ días & 7,0 $\pm 6,7$ días & 0,08 \\
\hline Total & $13,9 \pm 8,7$ días & $18,7 \pm 10,4$ días & 0,03 \\
\hline \multicolumn{4}{|l|}{ Cateterismo y reperfusión en SCA } \\
\hline Coronariografía & $88 \%$ & $64 \%$ & 0,038 \\
\hline ICP & $54 \%$ & $22 \%$ & 0,038 \\
\hline CRC & $4 \%$ & $7 \%$ & 0,53 \\
\hline Angioplastia primaria en SCACEST & $68 \%$ & $50 \%$ & 1 \\
\hline FSVI & & & 0,18 \\
\hline FSVI $>50 \%$ & $78 \%$ & $53 \%$ & \\
\hline FSVI $<30 \%$ & $6 \%$ & $10 \%$ & \\
\hline Técnicas especiales & & & 0,046 \\
\hline BIAC & $1,50 \%$ & $8 \%$ & 0,163 \\
\hline IOT & $4,50 \%$ & $17 \%$ & 0,07 \\
\hline TRS & $1,50 \%$ & $4 \%$ & 0,45 \\
\hline \multicolumn{4}{|l|}{ Tratamiento al alta } \\
\hline IECA o ARA II & $69 \%$ & $33 \%$ & 0,01 \\
\hline Espironolactona & $5 \%$ & $6 \%$ & 1 \\
\hline Diurético & $23 \%$ & $60 \%$ & 0,007 \\
\hline Muerte & $6 \%$ & $33 \%$ & 0,002 \\
\hline
\end{tabular}

ARA II: antagonistas del receptor de la angiotensina II; BIAC: balón de contrapulsación intraaórtico; CRC: cirugía de revascularización; DAFR: deterioro agudo de la función renal; FSVI: función sistólica del ventrículo izquierdo; IC: insuficiencia cardiaca; ICP: intervención coronaria percutánea; IECA: inhibidores de la enzima convertidora de angiotensina; IOT: intubación orotraqueal; SCA: síndrome coronario agudo; SCACEST: síndrome coronario agudo con elevación del ST; SCASEST: síndrome coronario agudo sin elevación del ST; TRS: terapia renal sustitutiva; UC: Unidad Coronaria.

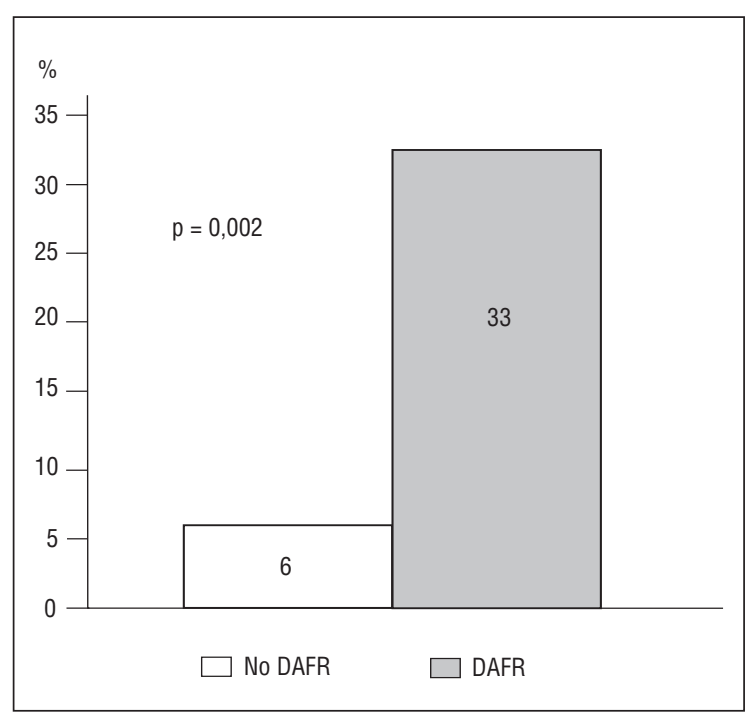

Figura 2. Mortalidad durante el ingreso. DAFR: deterioro agudo de la función renal.

a los criterios RIFLE ${ }^{16}$ de fracaso renal agudo. El paciente cardiópata agudo está particularmente expuesto al daño renal por causas hemodinámicas e iatrogénicas, y nuestro objetivo fue analizar si pequeños deterioros de la función renal tenían un impacto sobre el pronóstico a corto plazo. De hecho, con la definición empleada se obtuvo una incidencia de DAFR del $26 \%$, con una elevación media de la creatinina sérica de $1,5 \pm 0,9 \mathrm{mg} / \mathrm{dl}$ que ya tuvo un efecto significativo en la supervivencia durante el ingreso.
Los datos disponibles sobre la incidencia de deterioro de la función renal en pacientes cardiológicos agudos proceden en su mayoría de estudios extranjeros. En un metaanálisis ${ }^{13}$ de pacientes con SCA, la incidencia global de insuficiencia renal fue del $40 \%$, si bien se consideró el aclaramiento de creatinina inferior a $70 \mathrm{ml} / \mathrm{min}$ y se trató de una muestra más homogénea.

Estas cifras contrastan con la incidencia de fracaso renal agudo en una Unidad de Vigilancia Intensiva polivalente. En el estudio multicéntrico FRAMI ${ }^{17}$, realizado en nuestro medio, se recogieron 901 casos de fracaso renal agudo definido por el aumento de la creatinina $\geq 2 \mathrm{mg} / \mathrm{dl}$ o la diuresis $<400 \mathrm{ml}$ en 24 horas, lo que supuso una incidencia global del 5,7\%, similar a la hallada en registros internacionales ${ }^{14}$. $\mathrm{La}$ elevada incidencia de DAFR encontrada en nuestro estudio responde al uso de criterios diagnósticos más sensibles, pero también puede deberse a la situación de bajo gasto cardiaco y al amplio uso de fármacos y técnicas potencialmente nefrotóxicas en el cardiópata agudo.

Se ha demostrado que la insuficiencia renal constituye un potente predictor de muerte a corto y largo plazo en pacientes ingresados en la Unidad Coronaria $^{18}$. En el anterior metaanálisis ${ }^{13}$, los pacientes con SCA y función renal conservada presentaron una mortalidad aproximada del 5\%, frente al $10 \%$ de los pacientes con insuficiencia renal. En nuestra serie la diferencia en la mortalidad entre ambos grupos fue más llamativa, aunque este dato debe interpretarse con precaución por la distinta definición de insuficiencia renal, la heterogeneidad de la muestra y la posible presencia de factores de confusión. 
Los pacientes con DAFR tendieron a presentar un peor perfil de riesgo cardiovascular y significativamente más antecedentes de hipertensión arterial. La prevalencia ${ }^{15}$ de insuficiencia renal crónica en la población general es del 5-10\%. En nuestra serie la prevalencia global fue superior (18\%), y significativamente mayor en los pacientes con DAFR (45\%).

$\mathrm{Al}$ igual que en otros estudios ${ }^{19,20}$, los pacientes isquémicos agudos con insuficiencia renal recibieron significativamente menos cateterismos y procedimientos intervencionistas percutáneos. Esto puede deberse a sus características basales más adversas, a la indicación más restrictiva de cateterismos en pacientes nefrópatas por el riesgo de nefropatía inducida por contraste y al intento de estabilización médica inicial en pacientes que ingresan por un SCASEST en situación de insuficiencia cardiaca aguda grave, hecho que se relaciona frecuentemente con el deterioro de la función renal. Los pacientes con DAFR recibieron menos inhibidores de la enzima convertidora de angiotensina (IECA) al alta que los pacientes con función renal conservada. Existen datos convincentes ${ }^{21}$ que avalan el uso de IECA en insuficiencia cardiaca incluso en pacientes con insuficiencia renal avanzada, siempre que se inicien a dosis bajas y se titulen lentamente con especial vigilancia de las cifras de creatinina y potasio.

A pesar de las limitaciones del estudio, la heterogeneidad de la muestra, el diseño retrospectivo y el tamaño muestral, este trabajo ofrece una idea aproximada de cuál es la situación y el manejo actual de los pacientes cardiológicos agudos que desarrollan insuficiencia renal en nuestro medio. Nuestros hallazgos son superponibles a los de otros trabajos realizados a nivel internacional, el deterioro de la función renal es un proceso frecuente en pacientes hospitalizados en una Unidad de Cuidados Intensivos cardiológicos y su presencia se asocia a elevada morbimortalidad.

\section{Declaración de conflicto de intereses}

Los autores han declarado no tener ningún conflicto de intereses.

\section{BIBLIOGRAFÍA}

1. Boix R, Cañellas S, Almazán J, Cerrato E, Meseguer CM, Medrano MJ. Mortalidad cardiovascular en España. Año 2000. Boletín Epidemiológico Semanal. 2003;11:241-52.

2. Parfrey PS, Foley RN. The clinical epidemiology of cardiac disease in chronic renal failure. J Am Soc Nephrol. 1999;10:1606-15.

3. Bongartz LG, Cramer MJ, Doevendans PA, Joles JA, Braam B. The severe cardiorenal syndrome: "Guyton revisited". Eur Heart J. 2005;26:11-7.

4. Guías SEN. Riñón y enfermedad cardiovascular. Nefrologia. 2004;24:S1-235.
5. Herzog CA, Ma JZ, Collins AJ. Poor long-term survival after acute myocardial infarction among patients on long-term dialysis. N Eng J Med. 1998;339:799-805.

6. Schoebel FC, Gradaus F, Ivens K, Heering P, Jax TW, Grabensee B, et al. Reestenosis after elective coronary balloon angioplasty in patients with end stage renal disease: a case-control study using quantitative coronary angiography. Heart. 1997;78: $337-42$.

7. Iakovou I, Schmidt T, Bonizzoni E, Ge L, Sangiorgi GM, Stankovic G, et al. Incidence, predictors, and outcome of thrombosis after successful implantation of drug-eluting stents. JAMA. 2005;293:2126-30.

8. Herzog CA, Ma JZ, Collins AJ. Long-term outcome of dialysis patients in the United States with coronary revascularization procedures. Kidney Int. 1999;56:324-32.

9. Liaño F, Pascual J. Epidemiology of acute renal failure: a prospective, multicenter, community-based study. Madrid Acute Renal Failure Study Group. Kidney Int. 1996;50:811-8.

10. Rihal CS, Textor SC, Grill DE, Berger PB, Ting HH, Best $\mathrm{PJ}$, et al. Incidence and prognostic importance of acute renal failure after percutaneous coronary intervention. Circulation. 2002;105:2259-64.

11. Schrier RW. Role of diminished renal function in cardiovacular mortality. Marker or pathogenetic factor? J Am Coll Cardiol. 2006;47:1-8.

12. Anavekar NS, McMurray JJ, Velázquez EJ, Solomon SD, Kober L, Rouleau JL, et al. Relation between renal dysfunction and cardiovascular outcomes after myocardial infarction. N Engl J Med. 2004;351:1285-95.

13. Al Suwaidi J, Reddan DN, Williams K, Pieper KS, Harrington RA, Califf RM, et al. Prognostic implications of abnormalities in renal function in patients with acute coronary syndromes. Circulation. 2002;106:974-80.

14. Uchino S, Kellum JA, Bellomo R, Doig GS, Morimatsu H, Morgera S, et al. Acute renal failure in critically ill patients: a multinational, multicenter study. JAMA. 2005;294:813-8.

15. Simal F, Martin JC, Bellido J, Arzua D, Mena FJ, González I, et al. Prevalence of mild to moderate chronic kidney disease in the general population of Spain. Hortega study. Nefrologia. 2004; 24:329-37.

16. Bellomo R, Ronco C, Kellum JA, Mehta RL, Palevsky P; Acute Dialysis Quality Initiative workgroup. Acute renal failure definition, outcome measures, animal models, fluid therapy and information technology needs: the Second International Consensus Conference of the Acute Dialysis Quality Initiative (ADQI) Group. Crit Care. 2004;8:R204-12.

17. Herrera-Gutiérrez ME, Seller-Pérez G, Maynar-Moliner J, Sánchez-Izquierdo-Riera JA; Grupo de trabajo Estado actual del fracaso renal agudo y de las técnicas de reemplazo renal en UCI. Estudio FRAMI. Epidemiología del fracaso renal agudo en las UCI españolas. Estudio prospectivo multicéntrico FRAMI. Med Intensiva. 2006;30:260-7.

18. McCullough PA, Soman SS, Shah SS, Smith ST, Marks $\mathrm{KR}$, Yee J, et al. Risks associated with renal dysfunction in the coronary care unit. J Am Coll Cardiol. 2000;36:679-84.

19. Dumaine R, Collet JP, Tanguy ML, Mansencal N, DuboisRande JL, Henry P, et al. Prognostic significance of renal insufficiency in patients presenting with acute coronary syndrome (the Prospective Multicenter SYCOMORE study). Am J Cardiol. 2004; 94:1543-7.

20. Januzzi JL, Cannon CP, DiBattiste PM, Murphy S, Weintraub W, Braunwald E; TACTICS-TIMI 18 Investigators. Effects of renal insufficiency on early invasive management in patients with acute coronary syndromes (The TACTICS-TIMI 18 Trial). Am J Cardiol. 2002;90:1246-9.

21. Shlipak MG. Pharmacotherapy for heart failure in patients with renal insufficiency. Ann Intern Med. 2003;138:917-24. 